\title{
On the irrationality of polynomial Cantor series
}

\author{
by \\ Jaroslav Hančl (Ostrava) and Robert Tijdeman (Leiden) \\ Dedicated to Wolfgang M. Schmidt on the occasion of his 75th birthday
}

1. Introduction. Irrationality is a subject with many open questions. In 1979 Apéry [1] succeeded in showing that $\zeta(3)$ is irrational (cf. Beukers $[2]$ ), but the irrationality of $\zeta(5), \zeta(7), \zeta(9), \ldots$ is still open (cf. Zudilin [14]). Here $\zeta(s)=\sum_{n=1}^{\infty} n^{-s}$. In his paper Apéry observed that

$$
\zeta(3)=\frac{5}{2} \sum_{n=1}^{\infty} \frac{(-1)^{n-1}}{n^{3}\left(\begin{array}{c}
2 n \\
n
\end{array}\right)} .
$$

This sum equals

$$
\frac{5}{4} \sum_{N=1}^{\infty} \frac{1}{\prod_{n=2}^{N} Q(n)} \quad \text { where } \quad Q(x)=-\frac{2 x^{2}(2 x-1)}{(x-1)^{3}}
$$

and is the motivation to write this paper. However, we have a more modest goal and study sums

$$
S=\sum_{N=1}^{\infty} \frac{P(N)}{\prod_{n=1}^{N} Q(n)}
$$

with polynomials $P(x), Q(x) \in \mathbb{Q}[x]$ such that $Q(n) \in \mathbb{Z}$ for all $n \in \mathbb{Z}$ and not with rational functions $Q(x)$.

In 1869 Cantor [3] proved a first irrationality result for sums of the form $\sum_{N=1}^{\infty}\left(b_{N} / \prod_{n=1}^{N} a_{n}\right)$ where $\left\{a_{n}\right\}_{n=1}^{\infty}$ and $\left\{b_{n}\right\}_{n=1}^{\infty}$ are sequences of positive integers with $a_{n} \geq 2$ for all $n$. His result was generalised in various ways by Oppenheim [10] in 1954 and their results are the basis of the results in the present paper; see Theorem 2.1. Extensions of Oppenheim's results were given by Erdős and Strauss [4], [5], Hančl [6], Hančl and Tijdeman [7]-[9],

2000 Mathematics Subject Classification: Primary 11J72.

Key words and phrases: irrationality, infinite series, polynomials.

The first author supported by the grants no. 201/04/0381, 201/07/0191 and MSM6198898701. 
and Tijdeman and Yuan [13]. The authors investigated in [9] the case where $\left\{a_{n}\right\}_{n=1}^{\infty}$ is of the form $a_{n}=a n+b$ for fixed positive integer constants $a, b$. A survey on these and related results can be found in Tijdeman [12, Sec. 4-6]. The present paper is an extension of that part of [9] in which $a_{n}=a n+b, b_{n}=P(n)$ for all $n$ where $a, b \in \mathbb{Z}, P(x) \in \mathbb{Z}[x]$.

In Sections 2 and 3 we assume that $P(x) \in \mathbb{Q}[x]$. In Section 2 we provide a criterion for the rationality of $S$ in terms of polynomials which occur in some expansion of $P$ with respect to $Q$. This criterion is used in Section 3 to derive sufficient conditions on $P$ and $Q$ for the irrationality of $S$. Finally, in Theorem 4.1, we deal with the case that the numerators are integers which approximate values of a polynomial and we display the underlying principle of the paper in a more general form in Theorem 4.2.

2. An irrationality criterion for polynomial Cantor sums. We consider sums of the form

$$
S=\sum_{N=1}^{\infty} \frac{P(N)}{\prod_{n=1}^{N} Q(n)}
$$

where $P(x) \in \mathbb{Q}[x], Q(x) \in \mathbb{Q}[x], P \neq 0, Q(n) \in \mathbb{Z}$ for every integer $n$, the denominators do not vanish, and the sum converges. If $Q$ is a constant then $Q(n)$ is an integer $a$ for all $n$ and

$$
S=\sum_{N=0}^{\infty} \frac{P(N)}{a^{N+1}} \in \mathbb{Q}
$$

(cf. [12, Section 4.1]). In what follows, we assume that $Q$ is non-constant. We use the convention that an empty product equals 1 . Observe that for deciding about rationality of $S$ we may assume that $P$ has integer coefficients, since we can multiply $P$ and $S$ by the lowest common multiple of the denominators of the coefficients of $P$.

If $\operatorname{deg} P<\operatorname{deg} Q$, then the irrationality of $S$ is an immediate consequence of more general results of Oppenheim [10].

TheOREM 2.1 (Oppenheim). Let $\left\{a_{n}\right\}_{n=1}^{\infty}$ and $\left\{b_{n}\right\}_{n=1}^{\infty}$ be sequences of integers such that $a_{n}>1$ for all $n$ and $b_{n} / a_{n} \rightarrow 0$ as $n \rightarrow \infty$. Then

$$
S:=\sum_{N=1}^{\infty} \frac{b_{N}}{\prod_{n=1}^{N} a_{n}}
$$

is rational if and only if $b_{n}=0$ for $n>n_{0}$.

Proof. If there is an $n_{1}$ such that $b_{n}$ has the same sign for all $n>n_{1}$, then we apply Theorem 4 of [10] to obtain the conclusion. Otherwise we apply Theorem 8 of the same paper to prove the statement. 
Corollary 2.1. Let $P(x), Q(x) \in \mathbb{Q}[x], P \neq 0, \operatorname{deg} P<\operatorname{deg} Q$ be such that $Q(n) \in \mathbb{Z}, Q(n) \neq 0$ for all positive integers $n$. Then

$$
S=\sum_{N=1}^{\infty} \frac{P(N)}{\prod_{n=1}^{N} Q(n)} \notin \mathbb{Q} .
$$

Proof. Without loss of generality we may assume that $P(x) \in \mathbb{Z}[x]$ and apply Theorem 2.1.

Suppose that $\operatorname{deg} P \geq \operatorname{deg} Q$. We write

$$
P(x)=A_{0}(x)+A_{1}(x) Q(x)+\cdots+A_{t}(x) Q(x) Q(x-1) \cdots Q(x-t+1)
$$

with $A_{j}(x) \in \mathbb{Q}[x], \operatorname{deg} A_{j}<\operatorname{deg} Q$ for $j=0,1, \ldots, t$ and $A_{t} \neq 0$. Then

$$
\begin{aligned}
S & =\sum_{j=0}^{t} \sum_{N=1}^{\infty} \frac{A_{j}(N) Q(N) Q(N-1) \cdots Q(N-j+1)}{\prod_{n=1}^{N} Q(n)} \\
& =\sum_{j=0}^{t} \sum_{N=1}^{j} A_{j}(N) Q(0) Q(-1) \cdots Q(N-j+1)+\sum_{j=0}^{t} \sum_{N=j+1}^{\infty} \frac{A_{j}(N)}{\prod_{n=1}^{N-j} Q(n)} \\
& =\sum_{N=1}^{t} \sum_{j=N}^{t} A_{j}(N) Q(0) Q(-1) \cdots Q(N-j+1)+\sum_{j=0}^{t} \sum_{N=1}^{\infty} \frac{A_{j}(N+j)}{\prod_{n=1}^{N} Q(n)} .
\end{aligned}
$$

We conclude that

$$
\begin{aligned}
S= & \sum_{N=1}^{t} \sum_{j=0}^{t-N} A_{N+j}(N) Q(0) Q(-1) \cdots Q(-j+1) \\
& +\sum_{N=1}^{\infty} \frac{1}{\prod_{n=1}^{N} Q(n)} \sum_{j=0}^{t} A_{j}(N+j) .
\end{aligned}
$$

The first term on the right-hand side is a rational number. To the second term on the right-hand side we apply Corollary 2.1. As $\operatorname{deg}\left(\sum_{j=0}^{t} A_{j}(x+j)\right)$ $<\operatorname{deg}(Q(x)), S \in \mathbb{Q}$ if and only if the polynomial $\sum_{j=0}^{t} A_{j}(x+j)$ vanishes for all positive integer values of $x$, i.e. is identically zero. Together with Corollary 2.1 this yields the first part of the following general result.

Theorem 2.2. Let $P(x), Q(x) \in \mathbb{Q}[x], P \neq 0, Q$ non-constant, $Q(n) \in \mathbb{Z}$ for all positive integers $n$, such that

$$
S=\sum_{N=1}^{\infty} \frac{P(N)}{\prod_{n=1}^{N} Q(n)}
$$

is a well-defined real number. Define $t$ and $A_{0}(x), \ldots, A_{t}(x) \in \mathbb{Q}[x]$ by

$$
\begin{aligned}
P(x)= & A_{0}(x)+A_{1}(x) Q(x)+A_{2}(x) Q(x) Q(x-1) \\
& +\cdots+A_{t}(x) Q(x) Q(x-1) \cdots Q(x-t+1)
\end{aligned}
$$


with $\operatorname{deg} A_{j}<\operatorname{deg} Q$ for $j=0,1, \ldots, t$ and $A_{t} \neq 0$. Then

$$
S \in \mathbb{Q} \Leftrightarrow \sum_{j=0}^{t} A_{j}(x+j) \equiv 0 .
$$

Moreover, if $P(x), Q(x) \in \mathbb{Z}[x], Q$ is monic and $S \in \mathbb{Q}$, then $S \in \mathbb{Z}$.

Proof. The first statement has already been proved. Let $S \in \mathbb{Q}$, $P(x), Q(x) \in \mathbb{Z}[x]$ and $Q$ be monic. Then $A_{0}(x), \ldots, A_{t}(x) \in \mathbb{Z}[x]$. Hence the first term on the right-hand side of (2) is an integer. Using this we obtain, by $(3)$,

$$
S \in \mathbb{Q} \Rightarrow S \in \mathbb{Z}+\sum_{N=1}^{\infty} \frac{1}{\prod_{n=1}^{N} Q(n)} \sum_{j=0}^{t} A_{j}(N+j)=\mathbb{Z}+0=\mathbb{Z} .
$$

REMARK 2.1. If $Q$ is linear, then all the polynomials $A_{j}$ are rational constants. Hence $S \in \mathbb{Q} \Leftrightarrow \sum_{j=0}^{t} A_{j}=0$. It follows from a general theorem of Shidlovskiü [11] that if $\sum_{j=0}^{t} A_{j} \neq 0$, then $S$ is transcendental.

EXAMPLE 2.1. Let $P(x)=p_{4} x^{4}+p_{3} x^{3}+p_{2} x^{2}+p_{1} x+p_{0} \in \mathbb{Z}[x], Q(x)=$ $q_{2} x^{2}+q_{1} x+q_{0} \in \mathbb{Z}[x]$ with $q_{2} \neq 0$. Then $A_{2}(x)=p_{4} / q_{2}^{2}$,

$$
\begin{aligned}
A_{1}(x)= & \frac{1}{q_{2}^{3}}\left\{\left(p_{3} q_{2}^{2}-2 p_{4} q_{1} q_{2}+2 p_{4} q_{2}^{2}\right) x\right. \\
& \left.+\left(p_{2} q_{2}^{2}-p_{3} q_{1} q_{2}-2 p_{4} q_{0} q_{2}+p_{4} q_{1}^{2}+p_{4} q_{1} q_{2}-p_{4} q_{2}^{2}\right)\right\}, \\
A_{0}(x)= & \frac{1}{q_{2}^{3}}\left(p_{1} q_{2}^{3}-p_{2} q_{1} q_{2}^{2}-p_{3} q_{0} q_{2}^{2}+p_{3} q_{1}^{2} q_{2}+2 p_{4} q_{0} q_{1} q_{2}-p_{4} q_{1}^{3}\right) x \\
& +\frac{1}{q_{2}^{3}}\left(p_{0} q_{2}^{3}-p_{2} q_{0} q_{2}^{2}+p_{3} q_{0} q_{1} q_{2}+p_{4} q_{0}^{2} q_{2}-p_{4} q_{0} q_{1}^{2}\right) .
\end{aligned}
$$

Hence $S \in \mathbb{Q}$ if and only if

$$
\begin{aligned}
p_{1} q_{2}^{3}-p_{2} q_{1} q_{2}^{2}-p_{3} q_{0} q_{2}^{2}+p_{3} q_{1}^{2} q_{2}+p_{3} q_{2}^{2} & \\
& +2 p_{4} q_{0} q_{1} q_{2}-p_{4} q_{1}^{3}-2 p_{4} q_{1} q_{2}+2 p_{4} q_{2}^{2}=0
\end{aligned}
$$

and

$$
\begin{aligned}
& p_{0} q_{2}^{3}-p_{2} q_{0} q_{2}^{2}+p_{2} q_{2}^{2}+p_{3} q_{0} q_{1} q_{2}-p_{3} q_{1} q_{2}+p_{3} q_{2}^{2} \\
& \quad+p_{4} q_{0}^{2} q_{2}-p_{4} q_{0} q_{1}^{2}-2 p_{4} q_{0} q_{2}+p_{4} q_{1}^{2}-p_{4} q_{1} q_{2}+p_{4} q_{2}+p_{4} q_{2}^{2}=0 .
\end{aligned}
$$

Moreover, according to the proof of Theorem 2.2, if $S \in \mathbb{Q}$, then $q_{2}^{3} S \in \mathbb{Z}$.

The special case $p_{4}=q_{1}=0$ has been treated in [12]. The even more special case $p_{4}=p_{2}=p_{1}=q_{1}=0, p_{3} \neq 0$ has been dealt with in [8]. As mentioned in [12] the condition in [8] should be read as $c=1$ and $b+a d=0$ in accordance with the above conditions. 
REMARK 2.2. The case of an alternating sum

$$
\sum_{N=1}^{\infty}(-1)^{N} \frac{P(N)}{\prod_{n=1}^{N} Q(n)}
$$

can be reduced to the above sum by replacing $Q$ with $-Q$.

3. Some sufficient conditions for irrationality. In this section we give sufficient conditions for the irrationality of

$$
S=\sum_{N=1}^{\infty} \frac{P(N)}{\prod_{n=1}^{N} Q(n)}
$$

where $P(x), Q(x) \in \mathbb{Q}[x], Q$ non-constant such that $Q(n) \in \mathbb{Z}$ and $Q(n) \neq 0$ for all $n \in \mathbb{N}$. The following theorem will be our starting point.

Theorem 3.1. Let $P(x) \in \mathbb{Q}[x], P \neq 0, Q(x) \in \mathbb{Q}[x], Q$ non-constant and $Q(n) \in \mathbb{Z}, Q(n) \neq 0$ for all positive integers $n$. Let $\mathcal{R}$ be a class of polynomials which have integer values at integer points. Suppose that $Q$ has the property that if $R \in \mathcal{R}, R \neq 0$ then there exist $A(x), B(x) \in \mathbb{Q}[x]$ such that

$$
R(x)=A(x) Q(x)+B(x),
$$

$\operatorname{deg} B<\max (\operatorname{deg} Q, \operatorname{deg} R)$ and $A(x+1)+B(x) \in \mathcal{R}, A(x+1)+B(x) \not \equiv 0$. Then $P \in \mathcal{R}$ implies that

$$
S=\sum_{N=1}^{\infty} \frac{P(N)}{\prod_{n=1}^{N} Q(n)} \notin \mathbb{Q} .
$$

Proof. If $\operatorname{deg} P<\operatorname{deg} Q$, then we can apply Corollary 2.1. Suppose $\operatorname{deg} P \geq \operatorname{deg} Q$. By our assumption there exist $A(x), B(x) \in \mathbb{Q}[x]$ such that

$$
P(x)=A(x) Q(x)+B(x)
$$

with $\operatorname{deg} B<\max (\operatorname{deg} Q, \operatorname{deg} R)$ and $A(x+1)+B(x) \in \mathcal{R}, A(x+1)+B(x)$ $\not \equiv 0$. Furthermore

$$
S=\sum_{N=1}^{\infty} \frac{A(N) Q(N)+B(N)}{\prod_{n=1}^{N} Q(n)}=A(1)+\sum_{N=1}^{\infty} \frac{A(N+1)+B(N)}{\prod_{n=1}^{N} Q(n)} .
$$

If $\operatorname{deg}(A(x+1)+B(x))<\operatorname{deg} Q$, then we apply Corollary 2.1 to conclude that $S \notin \mathbb{Q}$ in view of $A(x+1)+B(x) \not \equiv 0$. If $\operatorname{deg}(A(x+1)+B(x)) \geq \operatorname{deg} Q$, then we apply the above procedure to $A(x+1)+B(x)$ in place of $P(x)$. After at most $\operatorname{deg} P$ iterations we will arrive at a situation where we can apply Corollary 2.1 to conclude that $S \notin \mathbb{Q}$. 
EXAMPLE 3.1. Suppose that $\mathcal{R}$ is the class of polynomials $\sum_{i=0}^{T} r_{i} x^{i} \in$ $\mathbb{Q}[x]$ with non-negative coefficients $r_{i}$ and $Q(x)=\sum_{i=0}^{M} c_{i} x^{i} \in \mathbb{Z}[x], c_{M}>0$, $c_{i}<0$ for $i=0,1, \ldots, M-1$. We shall show in Theorem 3.2 that if $P(x) \in \mathcal{R}$, $P \neq 0$, then $S \notin \mathbb{Q}$.

ExAmple 3.2. Suppose that $\mathcal{R}$ is the same class as in the previous example and $Q(x)=x^{2}+1$. We shall show in Theorem 3.3 that if $P(x) \in \mathcal{R}$, $P \neq 0$, then $S$ is rational if and only if $P(x)=x^{2}$.

EXAMPLE 3.3. Let $\mathcal{R}$ be the same class as in Example 3.1 and $Q(x)=$ $x^{3}-x^{2}+x-1$. We shall show in Theorem 3.4 that if $P(x) \in \mathcal{R}, P \neq 0$, then $S \notin \mathbb{Q}$.

EXAMPLE 3.4. Let $\mathcal{R}$ be the class of polynomials $P(x)$ such that $P(x)-P(0) \in \mathbb{Z}[x], P(0) \in \mathbb{Q} \backslash \mathbb{Z}$. Let $Q(x) \in \mathbb{Z}[x]$ be monic. Then $S \notin \mathbb{Q}$.

In the remainder of this section $\mathcal{R}$ denotes the class of polynomials $\sum_{i=0}^{T} r_{i} x^{i} \in \mathbb{Q}[x]$ with non-negative coefficients $r_{i}$. Observe that by considering $P\left(x+x_{0}\right)$ in place of $P(x)$ for a suitable integer $x_{0} \geq 0$ we can always secure that the coefficients of $P$ are non-negative. Of course we then have to consider the rationality of

$$
\begin{aligned}
S & =\sum_{N=1}^{\infty} \frac{P\left(N+x_{0}\right)}{\prod_{n=1}^{N} Q\left(n+x_{0}\right)} \\
& =\left(\prod_{n=1}^{x_{0}} Q(n)\right) \sum_{N=1}^{\infty} \frac{P(N)}{\prod_{n=1}^{N} Q(n)}-\sum_{N=1}^{x_{0}} P(N) \prod_{n=N+1}^{x_{0}} Q(n)
\end{aligned}
$$

so that $Q$ is translated by the same $x_{0}$.

In the next theorem we allow $Q$ to be the product of polynomials from a certain class.

Theorem 3.2. Let $P(x), Q(x) \in \mathbb{Q}[x], P(x)=\sum_{i=0}^{T} a_{i} x^{i}$ with $a_{T}>0$ and $a_{i} \geq 0$ for every $i=0,1, \ldots, T-1, Q(x)=\prod_{m=1}^{M} C_{m}(x)$ with $C_{m}(x)=$ $\sum_{k=0}^{K_{m}} c_{m, k} x^{k} \in \mathbb{R}[x]$ for $m=1, \ldots, M$ such that $c_{m, K_{m}}>0$, but $c_{m, k} \leq 0$ for $k=0,1, \ldots, K_{m}-1$. Assume that $Q(x)$ is non-constant and $Q(n) \in \mathbb{Z}$, $Q(n) \neq 0$ for every positive integer $n$. Then

$$
S=\sum_{N=1}^{\infty} \frac{P(N)}{\prod_{n=1}^{N} Q(n)} \notin \mathbb{Q} .
$$

Proof. If $\operatorname{deg} P<\operatorname{deg} Q$, then from Theorem 2.1 we conclude that $S \notin \mathbb{Q}$ (even without using the fact that $Q$ is a special product). 
Assume that $\operatorname{deg} P \geq \operatorname{deg} Q$. Then we obtain, by applying division with remainder,

$$
\begin{array}{rlrl}
P(x) & =A_{1}(x) C_{1}(x)+B_{1}(x), & \operatorname{deg} B_{1}<\operatorname{deg} A_{1}, \\
A_{1}(x) & =A_{2}(x) C_{2}(x)+B_{2}(x), & \operatorname{deg} B_{2}<\operatorname{deg} A_{2}, \\
& \vdots & \vdots & \\
A_{M-1}(x) & =A_{M}(x) C_{M}(x)+B_{M}(x), & \operatorname{deg} B_{M}<\operatorname{deg} A_{M} .
\end{array}
$$

Hence $P(x)=A(x) Q(x)+B(x)$ with $A(x)=A_{1}(x) \cdots A_{M}(x)$ and

$$
\begin{aligned}
B(x)= & B_{1}(x)+B_{2}(x) C_{1}(x) \\
& +B_{3}(x) C_{1}(x) C_{2}(x)+\cdots+B_{M}(x) C_{1}(x) \cdots C_{M-1}(x) .
\end{aligned}
$$

So $\operatorname{deg} B<K_{1}+\cdots+K_{M}=\operatorname{deg} Q$. Thus $A(x), B(x) \in \mathbb{Q}[x]$. Since $P(x) \in \mathcal{R}$, we find by induction on $m$ that $A_{m}(x)$ and $B_{m}(x)$ have nonnegative coefficients in view of

$$
x^{i}=\frac{1}{c_{i, M_{j}}} x^{i-M_{i}} C_{i}(x)+\sum_{k=0}^{M_{i}-1} \frac{-c_{i, k}}{c_{i, M_{j}}} x^{i+k-M_{i}} \quad\left(i=1, \ldots, M_{j}, j=1,2, \ldots\right),
$$

which is repeatedly used and yields non-negative coefficients. Therefore $A(x+1)+B(x)$ has non-negative coefficients as well. Thus $A(x+1)+B(x)$ $\in \mathcal{R}$. Moreover, since $A(x)$ is non-trivial and $A$ and $B$ have non-negative coefficients, $A(x+1)+B(x)$ is also non-trivial. As before,

$$
S=\sum_{N=1}^{\infty} \frac{A(N+1)+B(N)}{\prod_{n=1}^{N} Q(n)} .
$$

We now repeat the above argument with $P(x)$ replaced by $A(x+1)$ $+B(x)$. This polynomial has lower degree than $P(x)$. If $\operatorname{deg}(A(x+1)+B(x))$ $<\operatorname{deg} Q$, then we apply Corollary 2.1 to conclude $S \notin \mathbb{Q}$, otherwise we apply division with remainder to $A(x+1)+B(x)$ in place of $P(x)$. After at most $\operatorname{deg} P$ iterations we may apply Corollary 2.1 to conclude $S \notin \mathbb{Q}$.

EXAMPLE 3.5. For every positive integer $k$ the number

$$
\sum_{N=2}^{\infty} \frac{\left(N^{2}+N+1\right)^{k}}{\prod_{n=2}^{N}\left(n^{3}-2 n^{2}+1\right)}
$$

is irrational. Indeed we have $\left(x^{3}-2 x^{2}+1\right)=(x-1)\left(x^{2}-x-1\right)$.

Corollary 3.1. Let $P(x), Q(x) \in \mathbb{Q}[x]$ and $P(x)=\sum_{i=0}^{T} a_{i} x^{i}$. Suppose that $a_{T}>0$ and $a_{i} \geq 0$ for every $i=0,1, \ldots, T-1$. Let all roots of the polynomial $Q(x)$ be real, non-integral and non-negative. Assume that $Q(x)$ 
is non-constant and that $Q(n) \in \mathbb{Z}$ and $Q(n) \neq 0$ for all $n \in \mathbb{N}$. Then

$$
S=\sum_{N=1}^{\infty} \frac{P(N)}{\prod_{n=1}^{N} Q(n)} \notin \mathbb{Q} .
$$

EXAMPLE 3.6. For every positive integer $k$ the number

$$
\sum_{N=1}^{\infty} \frac{N^{k}}{\prod_{n=1}^{N}\left(n^{2}-3 n+1\right)}
$$

is irrational.

REMARK 3.1. The condition that the roots of $Q$ are non-integral is only needed to guarantee that the terms of the series are well-defined.

THEOREM 3.3. Let $d, k$ and $r$ be positive integers with $r d \leq k$ and let $P(x)=\sum_{i=0}^{T} a_{i} x^{i} \in \mathbb{Q}[x]$. Assume that $a_{T}>0$ and $a_{i} \geq 0$ for $i=$ $0,1, \ldots, T-1$. If

$$
S=\sum_{N=1}^{\infty} \frac{P(N)}{\prod_{n=1}^{N}\left(n^{k}+n^{k-d}+\cdots+n^{k-r d}\right)} \in \mathbb{Q}
$$

then $k=r d$. If $k=r d$ and $d>1$ then $S \in \mathbb{Q}$ if and only if $P(x)=$ $c\left(x^{k}+x^{k-d}+\cdots+x^{d}\right)$ where $c$ is a fixed positive integer.

Proof.

SteP 1: The case $T \geq k+d$. We write $Q(x)=x^{k}+x^{k-d}+\cdots+x^{k-r d}$ and

$$
\begin{aligned}
P(N)= & \sum_{i=0}^{k+d-1} a_{i} N^{i}+\sum_{i=k+d}^{T} a_{i} N^{i} \\
= & \sum_{i=0}^{k+d-1} a_{i} N^{i}+\sum_{i=k+d}^{T} a_{i}\left\{\left(N^{i-k}-N^{i-k-d}\right)\left(N^{k}+N^{k-d}+\cdots+N^{k-r d}\right)\right. \\
& \left.+N^{i-(r+1) d}\right\} \\
= & A(N)\left(N^{k}+N^{k-d}+\cdots+N^{k-r d}\right)+B(N)
\end{aligned}
$$

where

$$
A(x)=\sum_{i=k+d}^{T} a_{i} x^{i-k-d}\left(x^{d}-1\right), \quad B(x)=\sum_{i=0}^{k+d-1} a_{i} x^{i}+\sum_{i=k+d}^{T} x^{i-(r+1) d} .
$$

Hence

$$
A(x+1)=\sum_{i=k+d}^{T} a_{i}(x+1)^{i-k-d} \sum_{j=1}^{d}\left(\begin{array}{l}
d \\
j
\end{array}\right) x^{j}
$$


Since $a_{T}>0$ and $a_{i} \geq 0$ for $i \geq 0$, the coefficient of $x$ of $A(x+1)$ is positive. Furthermore,

$$
\begin{aligned}
S & =\sum_{N=1}^{\infty} \frac{P(N)}{\prod_{n=1}^{N} Q(n)}=\sum_{N=1}^{\infty}\left(\frac{A(N)}{\prod_{n=1}^{N-1} Q(n)}+\frac{B(N)}{\prod_{n=1}^{N} Q(n)}\right) \\
& =\sum_{N=1}^{\infty} \frac{A(N+1)+B(N)}{\prod_{n=1}^{N} Q(n)} .
\end{aligned}
$$

The degree of $A(x+1)+B(x)=P^{*}(x)$ is at most $T^{*} \leq \max (T-k$, $k+d-1, T-(r+1) d)<T$, its coefficients are non-negative integers and the coefficient of $x$ in $P^{*}(x)$ is positive. We may iterate this procedure with $P^{*}$ in place of $P$ and continue until $\operatorname{deg}\left(P^{*}\right)<k+d$. From now on we assume that the conditions of Theorem 3.3 are satisfied with $T<k+d$ and remember that the coefficient of $x$ is positive if we have applied Step 1.

SteP 2: The case $T<k+d$. If $T<k$, then it follows immediately from Corollary 2.1 that $S \notin \mathbb{Q}$ if we have not applied Step 1 . However, if we have applied Step 1 , then the coefficient of $x$ is positive and also we have $S \notin \mathbb{Q}$. Therefore we suppose $k \leq T<k+d$. We write $P(x)=\sum_{i=0}^{T} a_{i} x^{i}$ for the present numerator of $S$. Hence

$$
\begin{aligned}
P(N)= & \sum_{i=0}^{k-1} a_{i} N^{i}+\sum_{i=k}^{T} a_{i} N^{i} \\
= & \sum_{i=0}^{k-1} a_{i} N^{i}+\sum_{i=k}^{T} a_{i} N^{i-k}\left(N^{k}+N^{k-d}+\cdots+N^{k-r d}\right) \\
& -\sum_{i=k}^{T} a_{i}\left(N^{i-d}+\cdots+N^{i-r d}\right) \\
= & A(N)\left(N^{k}+N^{k-d}+\cdots+N^{k-r d}\right)+B(N)
\end{aligned}
$$

where

$$
A(x)=\sum_{i=k}^{T} a_{i} x^{i-k}, \quad B(x)=\sum_{i=0}^{k-1} a_{i} x^{i}-\sum_{i=k}^{T} a_{i}\left(x^{i-d}+\cdots+x^{i-r d}\right) .
$$

Hence

$$
A(x+1)=\sum_{i=0}^{T-k} a_{i+k}(x+1)^{i}
$$

has positive coefficients in view of $a_{T}>0, a_{i} \geq 0$ for $i=k, \ldots, T$, and

$$
S=\sum_{N=1}^{\infty} \frac{A(N+1)+B(N)}{\prod_{n=1}^{N} Q(n)}
$$


where $P^{*}(x):=A(x+1)+B(x)$ has rational coefficients and degree $<k$. We distinguish between two cases.

CASE 2A: Let $k>r d$. Then we have $P^{*}(0)=\sum_{i=0}^{T-k} a_{i+k}+a_{0} \geq a_{T}>0$ so that $P^{*}$ is non-trivial. By Corollary 2.1 we obtain $S \notin \mathbb{Q}$.

CASE 2B: Let $k=r d$ and $d>1$. Then we have $P^{*}(0)=\sum_{i=0}^{T-k} a_{i+k}+a_{0}$ and this is positive if $T>k$. Thus $S \notin \mathbb{Q}$ if $T>k$ by Corollary 2.1. Suppose $T=k$. Then the coefficient of $x$ of $P^{*}(x)$ equals $a_{1}$ because of $T=k=r d$ and $d>1$. If $a_{1}>0$, then we know that $S \notin \mathbb{Q}$ by Corollary 2.1. This is certainly the case if we have applied Step 1. We conclude that $a_{1}=0$ is only possible if the original $T$ equals $k$. Then $A(x)=a_{k}$ and $B(x)=\sum_{i=0}^{k-1} a_{i} x^{i}-a_{k}\left(x^{k-d}+x^{k-2 d}+\cdots+1\right)$. According to Corollary 2.1, $S \in \mathbb{Q}$ if and only if $A(x+1)+B(x) \equiv 0$. Hence $S \in \mathbb{Q}$ if and only if

$$
\begin{aligned}
P(x) & =\sum_{i=0}^{k} a_{i} x^{i}=a_{k} x^{k}+a_{k}\left(x^{k-d}+x^{k-2 d}+\cdots+1\right)-a_{k} \\
& =a_{k}\left(x^{k}+x^{k-d}+\cdots+x^{d}\right) .
\end{aligned}
$$

This completes the proof of the theorem.

Example 3.7. Let $T, k \in \mathbb{N}$. Then

$$
\sum_{N=1}^{\infty} \frac{N^{T}}{\prod_{n=1}^{N}\left(n^{k}+n^{k-1}+\cdots+n\right)} \notin \mathbb{Q} .
$$

EXAMPlE 3.8. Let $T \in \mathbb{N}$. By applying Theorem 3.3 with $P(x)=x^{T}$ and $Q(x)=\frac{1}{2}\left(x^{k}+x^{k-1}\right)$ we obtain

$$
\sum_{N=1}^{\infty} \frac{2^{N} N^{T}}{\prod_{n=1}^{N}\left(n^{k}+n^{k-1}\right)} \notin \mathbb{Q} .
$$

REMARK 3.2. The following example shows that we cannot omit the condition $d>1$ if $k=r d$ in Theorem 3.3:

$$
\begin{aligned}
\sum_{N=1}^{\infty} & \frac{N^{5}+2 N^{3}+N^{2}}{\prod_{n=1}^{N}\left(n^{3}+n^{2}+n+1\right)} \\
& =\sum_{N=1}^{\infty} \frac{\left(N^{2}-N+2\right)\left(N^{3}+N^{2}+N+1\right)-N^{2}-N-2}{\prod_{n=1}^{N}\left(n^{3}+n^{2}+n+1\right)} \\
& =\sum_{N=1}^{\infty}\left(\frac{N^{2}-N+2}{\prod_{n=1}^{N-1}\left(n^{3}+n^{2}+n+1\right)}-\frac{N^{2}+N+2}{\prod_{n=1}^{N}\left(n^{3}+n^{2}+n+1\right)}\right)=1 \in \mathbb{Q} .
\end{aligned}
$$


THeOREM 3.4. Let $d, k$ and $r$ be positive integers with $r d \leq k$ and let $P(x)=\sum_{i=0}^{T} a_{i} x^{i} \in \mathbb{Q}[x]$. Assume that $a_{T}>0$ and $a_{i} \geq 0$ for $i=$ $0,1, \ldots, T-1$. Then

$$
S=\sum_{N=2}^{\infty} \frac{P(N)}{\prod_{n=2}^{N}\left(n^{k}-n^{k-d}+\cdots+(-1)^{r} n^{k-r d}\right)} \notin \mathbb{Q} .
$$

Proof. Let $T<k$. Then we apply Corollary 2.1 to conclude $S \notin \mathbb{Q}$. Assume that $T \geq k$. Now the proof splits into two cases.

CASE 1: Suppose that $r$ is odd. If $T \geq k+d$ then we use

$$
\begin{aligned}
x^{i} & =x^{i-k-d}\left(x^{k+d}-x^{k-r d}\right)+x^{i-(r+1) d} \\
& =x^{i-k-d}\left(x^{d}+1\right)\left(x^{k}-x^{k-d}+\cdots-x^{k-r d}\right)+x^{i-(r+1) d}
\end{aligned}
$$

to write

$$
P(x)=A(x)\left(x^{k}-x^{k-d}+\cdots-x^{k-r d}\right)+B(x)
$$

where $A(x), B(x) \in \mathbb{Q}[x], \operatorname{deg} B<T, \operatorname{deg} A \leq T-k, A(x)+B(x) \not \equiv 0$ and the polynomials $A, B$ have non-negative coefficients. Hence $A(x+1)+B(x) \not \equiv 0$ and, as before,

$$
S=q+\sum_{N=2}^{\infty} \frac{A(N+1)+B(N)}{\prod_{n=2}^{N}\left(n^{k}-n^{k-d}+\cdots-n^{k-r d}\right)}
$$

where $q$ is some rational number. We iterate this procedure with $P(x)$ replaced by $A(x+1)+B(x)$ until we get

$$
S=q^{*}+\sum_{N=2}^{\infty} \frac{P^{*}(N)}{\prod_{n=2}^{N}\left(n^{k}-n^{k-d}+\cdots-n^{k-r d}\right)}
$$

where $P^{*}(x) \in \mathbb{Q}[x], P^{*} \neq 0, T^{*}:=\operatorname{deg}\left(P^{*}\right)<k+d$ and all coefficients of $P^{*}$ are non-negative.

For $k \leq T^{*}<k+d$ we use

$$
\begin{aligned}
x^{i}= & x^{i-k}\left(x^{k}-x^{k-d}+x^{k-2 d}-\cdots-x^{k-r d}\right) \\
& +x^{i-d}-x^{i-2 d}+x^{i-3 d}+\cdots+x^{i-r d}
\end{aligned}
$$

to write

$$
P^{*}(x)=A^{*}(x)\left(x^{k}-x^{k-d}+\cdots-x^{k-r d}\right)+B^{*}(x)
$$

where $A^{*}(x), B^{*}(x) \in \mathbb{Q}[x], \operatorname{deg}\left(B^{*}\right)<k, \operatorname{deg}\left(A^{*}\right)<d, B^{*} \neq 0$. The polynomial $A^{*}$ has non-negative coefficients. Since $A^{*}(1)+B^{*}(0) \neq 0$, we have $A^{*}(x+1)+B^{*}(x) \not \equiv 0$. In this way we get a representation

$$
S=q^{* *}+\sum_{N=2}^{\infty} \frac{P^{* *}(N)}{\prod_{n=2}^{N}\left(n^{k}-n^{k-d}+\cdots-n^{k-r d}\right)}
$$


where $q^{* *}$ is some rational number, $P^{* *}(x):=A^{*}(x+1)+B^{*}(x) \not \equiv 0$ and $\operatorname{deg}\left(P^{* *}\right)<k$. We apply Corollary 2.1 to conclude $S \notin \mathbb{Q}$.

CASE 2: Suppose that $r$ is even. If $T \geq k+(r+2) d$ then we use $x^{i}=x^{i-k-(r+2) d}\left(x^{d}+1\right)\left(x^{k}-x^{k-d}+\cdots+x^{k-r d}\right)\left(x^{(r+1) d}-1\right)+x^{i-2(r+1) d}$ to obtain

$$
P(x)=: A_{1}(x)\left(x^{k}-x^{k-d}+\cdots+x^{k-r d}\right)+B_{1}(x)
$$

where $A_{1}(x+1), B_{1}(x) \in \mathbb{Q}[x], \operatorname{deg} B<T, \operatorname{deg} A_{1} \leq T-k, A_{1}(x+1)+B_{1}(x)$ $\not \equiv 0$ and the polynomials $A_{1}(x+1), B_{1}(x)$ have non-negative coefficients. As in the first case we get

$$
S=q_{0}+\sum_{N=2}^{\infty} \frac{A_{1}(N+1)+B_{1}(N)}{\prod_{n=2}^{N}\left(n^{k}-n^{k-d}+\cdots+n^{k-r d}\right)}
$$

where $q_{0}$ is some rational number. We iterate this procedure until we obtain

$$
S=q_{1}+\sum_{N=2}^{\infty} \frac{P_{1}(N)}{\prod_{n=2}^{N}\left(n^{k}-n^{k-d}+\cdots+n^{k-r d}\right)}
$$

where $q_{1} \in \mathbb{Q}, P_{1}(x) \in \mathbb{Q}[x], P_{1} \neq 0, \operatorname{deg} P_{1}<k+(r+2) d$ and all coefficients of $P_{1}$ are non-negative. Let $T_{1}$ be the degree of $P_{1}$.

If $k+(r+1) d \leq T_{1}<k+(r+2) d$ then we use

$$
\begin{aligned}
x^{i}= & \left(x^{i-k}+x^{i-k-d}-x^{i-k-(r+1) d}\right)\left(x^{k}-x^{k-d}+\cdots+x^{k-r d}\right) \\
& -x^{i-(r+2) d}+x^{i-(r+3) d}-\cdots+x^{i-(2 r+1) d} \\
= & A_{2}(x)\left(x^{k}-x^{k-d}+\cdots+x^{k-r d}\right)+B_{2}(x)
\end{aligned}
$$

where $A_{2}(x)=x^{i-k}+x^{i-k-d}-x^{-k-(r+1) d}$ and $B_{2}(x)=-x^{i-(r+2) d}+$ $x^{i-(r+3) d}-\cdots+x^{i-(2 r+1) d}$. The polynomial $A_{2}(x+1)=(x+1)^{i-k}+$ $(x+1)^{i-k-d}-(x+1)^{i-k-(r+1) d}=(x+1)^{i-k-(r+1) d}\left((x+1)^{(r+1) d}+(x+1)^{r d}-1\right)$ has positive coefficients and $\operatorname{deg} B_{2}<k$. Therefore the coefficients in the polynomial $A_{2}(x+1)+B_{2}(x)$ of powers $x^{m}$ with $m \geq k$ are all non-negative and $A_{2}(1)+B_{2}(0)>0$. In this way we obtain a representation

$$
S=q_{2}+\sum_{N=2}^{\infty} \frac{P_{2}(N)}{\prod_{n=2}^{N}\left(n^{k}-n^{k-d}+\cdots+n^{k-r d}\right)}
$$

with $q_{2} \in \mathbb{Q}, P_{2}(x) \in \mathbb{Q}[x], \operatorname{deg} P_{2}<k+(r+1) d$ and all the coefficients of $x^{m}$ in $P_{2}$ with $m \geq k$ non-negative and $P_{2}(0)>0$. Let $T_{2}$ be the degree of $P_{2}$.

If $k+d \leq T_{2}<k+(r+1) d$ then we use

$$
\begin{aligned}
x^{i} & =\left(x^{i-k}+x^{i-k-d}\right)\left(x^{k}-x^{k-d}+\cdots+x^{k-r d}\right)-x^{i-(r+1) d} \\
& =A_{3}(x)\left(x^{k}-x^{k-d}+\cdots+x^{k-r d}\right)+B_{3}(x)
\end{aligned}
$$


where $A_{3}(x)=x^{i-k}+x^{i-k-d}$ and $B_{3}(x)=-x^{i-(r+1) d}$. The coefficients in the polynomial $A_{3}(x+1)+B_{3}(x)=(x+1)^{i-k}+(x+1)^{i-k-d}-x^{i-(r+1) d}$ of powers $x^{m}$ with $m \geq k$ are all non-negative and $A_{3}(1)+B_{3}(0)>0$. In this way we obtain a representation

$$
S=q_{3}+\sum_{N=2}^{\infty} \frac{P_{3}(N)}{\prod_{n=2}^{N}\left(n^{k}-n^{k-d}+\cdots+n^{k-r d}\right)}
$$

with $q_{3} \in \mathbb{Q}, P_{3}(x) \in \mathbb{Q}[x], \operatorname{deg} P_{3}<k+d$ and all the coefficients of $x^{m}$ in $P_{3}$ with $m \geq k$ non-negative and $P_{3}(0)>0$. Let $T_{3}$ be the degree of $P_{3}$.

Next, if $k \leq T_{3}<k+d$, then we use

$$
\begin{aligned}
x^{i} & =x^{i-k}\left(x^{k}-x^{k-d}+\cdots+x^{k-r d}\right)+x^{i-d}-x^{i-2 d}+\cdots+x^{i-(r-1) d}-x^{i-r d} \\
& =A_{4}(x)\left(x^{k}-x^{k-d}+\cdots+x^{k-r d}\right)+B_{4}(x)
\end{aligned}
$$

where $A_{4}(x)=x^{i-k}$ and $B_{4}(x)=x^{i-d}-x^{i-2 d}+\cdots+x^{i-(r-1) d}-x^{i-r d}$. Hence $A_{4}(x+1)+B_{4}(x)=(x+1)^{i-k}+x^{i-d}-x^{i-2 d}+\cdots+x^{i-(r-1) d}-x^{i-r d}$ satisfies $\operatorname{deg}\left(A_{4}(x+1)+B_{4}(x)\right)<k$ and $A_{4}(1)+B_{4}(0)>0$. This leads to

$$
S=q_{4}+\sum_{N=2}^{\infty} \frac{P_{4}(N)}{\prod_{n=2}^{N}\left(n^{k}-n^{k-d}+\cdots+n^{k-r d}\right)}
$$

where $q_{4} \in \mathbb{Q}, P_{4}(x) \in \mathbb{Q}[x], \operatorname{deg} P_{4}<k$ and $P_{4} \neq 0$. Finally we apply Corollary 2.1 to conclude $S \notin \mathbb{Q}$.

EXAMPLE 3.9. As an immediate consequence of Theorem 3.4 we obtain

$$
\sum_{N=2}^{\infty} \frac{(3 N+5)^{7}+(3 N-5)^{7}}{\prod_{n=1}^{N}\left(n^{5}-n^{4}+n^{3}-n^{2}+n-1\right)} \notin \mathbb{Q} .
$$

EXAMPLE 3.10. Let $a_{1}, b_{1}, c_{1}, a_{2}, b_{2}, c_{2}$ and $d$ be integers with $a_{1} \geq\left|a_{2}\right|$, $b_{1} \geq\left|b_{2}\right|, c_{1} \geq\left|c_{2}\right|$ and $d>0$. Then, by Theorem 3.4,

$$
\sum_{N=2}^{\infty} \frac{\left(a_{1} N^{2}+b_{1} N+c_{1}\right)^{d}+\left(a_{2} N^{2}+b_{2} N+c_{2}\right)^{d}}{\prod_{n=1}^{N}\left(n^{4}-n^{3}+n^{2}-n+1\right)} \in \mathbb{Q}
$$

if and only if $a_{1}=-a_{2}, b_{1}=-b_{2}, c_{1}=-c_{2}$ and $d$ is odd.

4. Some variations. In this section we present two theorems which deal with sums related to those studied in the previous sections. In the first theorem the numerator attains integer values, but behaves approximately like a polynomial.

TheOREM 4.1. Let $P(x), Q(x) \in \mathbb{Q}[x]$ be such that $Q$ is non-constant and $Q(n) \in \mathbb{Z}, Q(n) \neq 0$ for all positive integers $n$. Let $f: \mathbb{N} \rightarrow \mathbb{Z}$ 
be a sequence such that $f(N)=P(N)+o(Q(N))$ as $N \rightarrow \infty$. Define $A_{0}(x), \ldots, A_{t}(x) \in \mathbb{Q}[x]$ by (1). If

$$
S=\sum_{N=1}^{\infty} \frac{f(N)}{\prod_{n=1}^{N} Q(n)} \in \mathbb{Q},
$$

then there exists $N_{0}$ such that

$$
f(N)=P(N)-\sum_{j=0}^{t} A_{j}(N+j) \quad \text { for } N>N_{0} .
$$

Proof. Let $A$ be a common denominator of the coefficients of $A_{0}, A_{1}, \ldots$, $A_{t}, P$. Then, by (1) and (2),

$$
\begin{aligned}
A \sum_{N=1}^{\infty} \frac{f(N)}{\prod_{n=1}^{N} Q(n)} & \\
= & A \sum_{N=1}^{\infty} \frac{P(N)}{\prod_{n=1}^{N} Q(n)}+A \sum_{N=1}^{\infty} \frac{f(N)-P(N)}{\prod_{n=1}^{N} Q(n)} \\
= & \sum_{N=1}^{t} \sum_{j=0}^{t-N} A A_{N+j}(N) Q(0) Q(-1) \cdots Q(-j+1) \\
& +\sum_{N=1}^{\infty} \frac{A}{\prod_{n=1}^{N} Q(n)} \sum_{j=0}^{t} A_{j}(N+j)+A \sum_{N=1}^{\infty} \frac{f(N)-P(N)}{\prod_{n=1}^{N} Q(n)} \\
\in & \mathbb{Z}+\sum_{N=1}^{\infty} \frac{A \sum_{j=0}^{t} A_{j}(N+j)+A(f(N)-P(N))}{\prod_{n=1}^{N} Q(n)} .
\end{aligned}
$$

The numerator of the fraction is, for every positive integer $N$, an integer which is $o(Q(N))$ as $N \rightarrow \infty$. Hence, by Theorem 4 of Oppenheim [10] if the denominator has ultimately a fixed sign and by Theorem 8 of the same paper otherwise, the infinite sum is rational if and only if $\sum_{j=0}^{t} A_{j}(N+j)$ $+f(N)-P(N)=0$ for $N>N_{0}$. Thus $S \in \mathbb{Q}$ if and only if (4) holds.

Remark 4.1. Note that $\sum_{j=0}^{t} A_{j}(x+j) \in \mathbb{Q}[x]$ so that, for $N$ large, $f(N)$ has to be the value at $N$ of a polynomial $f(x) \in \mathbb{Z}[x]$.

REMARK 4.2. By adjusting the method from Hančl and Tijdeman [9] it can be proved that if in the above theorem it is only assumed that $P(x) \in \mathbb{R}[x]$, then $S \in \mathbb{Q}$ implies that $P(x) \in \mathbb{Q}[x]$.

EXAmPle 4.1. By applying Theorem 4.1 with $P(x)=x^{3}, Q(x)=x^{2}+1$, we obtain

$$
\sum_{N=1}^{\infty} \frac{\left[N^{3}-\frac{1}{2} N\right]}{\prod_{n=1}^{N}\left(n^{2}+1\right)} \notin \mathbb{Q}, \quad \sum_{N=1}^{\infty} \frac{\left[N^{3}-N \sqrt{2}\right]}{\prod_{n=1}^{N}\left(n^{2}+1\right)} \notin \mathbb{Q} .
$$


TheOREM 4.2. Let $t \in \mathbb{N}$. Let $\left\{a_{n}\right\}_{n=-t}^{\infty}$ and $\left\{b_{n}\right\}_{n=1}^{\infty}$ be two integer sequences such that $a_{n}>1$ and $b_{n}=\sum_{j=0}^{t} b_{n, j} a_{n} a_{n-1} \cdots a_{n-j+1}$ for $n=$ $1,2, \ldots$, where the $b_{n, j}$ are integers and $\sum_{j=0}^{t} b_{n+j, j}=o\left(a_{n}\right)$ as $n \rightarrow \infty$. Then

$$
S=\sum_{N=1}^{\infty} \frac{b_{N}}{\prod_{n=1}^{N} a_{n}} \in \mathbb{Q}
$$

if and only if $\sum_{j=0}^{t} b_{N+j, j}=0$ for all $N$ larger than some $N_{0}$.

Proof. We have, similarly to the derivation of (2),

$$
\begin{aligned}
S & =\sum_{N=1}^{\infty} \frac{\sum_{j=0}^{t} b_{N, j} a_{N} \cdots a_{N-j+1}}{\prod_{n=1}^{N} a_{n}} \\
& =\sum_{j=1}^{t} \sum_{N=1}^{j} b_{N, j} a_{0} a_{-1} \cdots a_{N-j+1}+\sum_{j=0}^{t} \sum_{N=j+1}^{\infty} \frac{b_{N, j}}{\prod_{n=1}^{N-j} a_{n}} \\
& =\sum_{N=1}^{t} \sum_{j=0}^{t-N} b_{N, N+j} a_{0} a_{-1} \cdots a_{-j+1}+\sum_{N=1}^{\infty} \frac{1}{\prod_{n=1}^{N} a_{n}} \sum_{j=0}^{t} b_{N+j, j} .
\end{aligned}
$$

The first term on the right-hand side is a rational integer. Suppose $\sum_{j=0}^{t} b_{N+j, j}=o\left(a_{N}\right)$ as $N \rightarrow \infty$. Then by Oppenheim's Theorems 4 and 8 of [10], $S \in \mathbb{Q}$ if and only if $\sum_{j=0}^{t} b_{N+j, j}=0$ for $N>N_{0}$.

Corollary 4.1. Suppose $b_{n, j} \geq 0$ for all $n$ and $j$ in Theorem 4.2. If $\sum_{j=0}^{t} b_{n+j, j}=o\left(a_{n}\right)$ as $n \rightarrow \infty$, then $S \in \mathbb{Q}$ if and only if $b_{n}=0$ for all $n$ larger than some $n_{0}$.

ExAmple 4.2. Let $k \in \mathbb{N}$ and $d(n)$ be the number of divisors of $n$. Then

$$
\sum_{N=2}^{\infty} \frac{d(N) N^{k}}{\prod_{n=2}^{N}(n-d(n))} \notin \mathbb{Q} .
$$

\section{References}

[1] R. Apéry, Irrationalité de $\zeta 2$ et $\zeta 3$, Journ. Arithm. 1978, Astérisque 61 (1979), $11-13$.

[2] F. Beukers, A note on the irrationality of $\zeta(2)$ and $\zeta(3)$, Bull. London Math. Soc. 11 (1979), 268-272.

[3] G. Cantor, Über die einfachen Zahlensysteme, Z. Math. Phys. Jahrg. 14 (1869), 121-128; Coll. Papers, Springer, Berlin, 1932, 35-42.

[4] P. Erdős and E. G. Straus, Some number theoretic results, Pacific J. Math. 36 (1971), 635-646.

[5] - - - On the irrationality of certain series, ibid. 55 (1974), 85-92.

[6] J. Hančl, A note on a paper of Oppenheim and Šalát concerning series of Cantor type, Acta Math. Inform. Univ. Ostraviensis 10 (2002), 35-41. 
[7] J. Hančl and R. Tijdeman, On the irrationality of Cantor series, J. Reine Angew. Math. 571 (2004), 145-158.

[8] - - -, On the irrationality of Cantor and Ahmes series, Publ. Math. Debrecen 65 (2004), 371-380.

[9] —, - On the irrationality of factorial series, Acta Arith. 118 (2005), 383-401.

[10] A. Oppenheim, Criteria for irrationality of certain classes of numbers, Amer. Math. Monthly 61 (1954), 235-241.

[11] A. B. Shidlovskiŭ, On the transcendence and algebraic independence of the values of certain functions, Trudy Moskov. Mat. Obshch. 8 (1959), 283-320 (in Russian); English transl.: Amer. Math. Soc. Transl. Ser. 227 (1963), 191-230.

[12] R. Tijdeman, On irrationality and transcendency of infinite sums of rational numbers, in: Diophantine Equations, TIFR, Mumbai, to appear.

[13] R. Tijdeman and P. Z. Yuan, On the rationality of Cantor and Ahmes series, Indag. Math. (N.S.) 13 (2002), 407-418.

[14] V. V. Zudilin, On the irrationality of $\zeta_{q}(2)$, Uspekhi Mat. Nauk 56 (2001), no. 6, 147-148 (in Russian); English transl.: Russian Math. Surveys 56 (2001), 1183-1185.

Department of Mathematics

and Institute for Research and Applications

of Fuzzy Modeling

University of Ostrava

30. dubna 22

70103 Ostrava 1, Czech Republic

E-mail: hancl@osu.cz
Mathematisch Instituut

Universiteit Leiden

Niels Bohrweg 1

Postbus 9512

2300 RA Leiden

The Netherlands

E-mail: tijdeman@math.leidenuniv.nl

Received on 7.3.2007

and in revised form on 9.8.2007 